\title{
French and Slovak brand names - contrastive aspect
}

\section{[Les noms de marques francais et slovaques, aspect contrastif]}

\author{
Ludmila Meskova - Jana Pecnikova
}

DOI: 10.18355/XL.2019.12.01XL.16

\begin{abstract}
The paper is focused on the issue of brand names from a linguistic point of view. It deals with the compilation of brand name database in French and Slovak language and their semantic and formal analysis to point out differences among them. Specifically, discussion forums and blogs are the sources of the excerpting of brand names. The brands are lexicalized, and they become common nouns (Škoda: škodovka, Coca Cola: kola, Botas: botasky, Champagne: šampanské, Cognac: koňak, etc.). The nouns of brands can be understandable for native speakers, but it is not the case of foreigners. Many brands are typical of various cultures, but some of them are only typical for a specific culture. The creation of brands is accompanied by lexical creativity.
\end{abstract}

Key words: brand names, derivations of brand names, French-Slovak database of brand names, cultural identity, lexical creativity

\section{Résumé}

L'objectif de cet article est orienté vers l'aspect linguistique des noms de marques. Il s'agit d'élaborer une base de données franco-slovaque des noms de marques intégrés à la langue courante, de les analyser sémantiquement et formellement et d'identifier les différences éventuelles entre les deux langues. Le corpus présenté ici, servant à répertorier des noms de marques, a été constitué pour l'essentiel à partir de forums de spécialistes et de blogs. Les noms de marques entrent dans le lexique général (Škoda: škodovka, Coca Cola: kola, Botas: botasky, etc.). Certains noms de marques naturellement compréhensibles pour les locuteurs natifs, peuvent paraître opaques aux locuteurs étrangers. De nombreux noms de marques sont communes à plusieurs cultures, d'autres sont profondément inscrits dans un contexte particulier. La création des noms de marques est caractérisée par la création lexicale.

Mots clés : noms de marques, dérivés des noms de marques, base de données francoslovaque des noms de marques, identité culturelle, créativité lexicale

\section{Introduction}

La problématique des noms de marques en français a attiré notre attention. Inspirées par nos collègues français ${ }^{1}$, nous avons eu l'idée d'étudier ce phénomène en slovaque. L'objectif de cette recherche est d'élaborer une base de données franco-slovaque des noms de marques et dérivés intégrés à la langue courante, de les analyser et d'identifier les différences éventuelles entre les deux langues. Nous ne prendrons pas en compte l'aspect marketing et centrerons notre problématique uniquement sur les

\footnotetext{
${ }^{1}$ En coopération avec Gabrielle Le Tallec Lloret et son équipe, nous avons monté un projet APVV SK-FR 2017-0018 / PHC Stefanik 2018 LENOMA concernant l'étude et la comparaison des noms de marques en français et en slovaque. Ce projet a été financé par l'Agence slovaque pour le soutien à la recherche et au développement. L'objectif collaboratif du projet est d'étendre à la langue slovaque le travail en cours sur la base de données (anglais, français, italien) existant déjà entre l'Université Paris 13 (G. Le Tallec-Lloret) et l'Université L'Orientale de Naples (Jana Altmanova).
} 
questions linguistiques et culturelles du thème de recherche. Nous aimerions susciter l'intérêt des francophones comme des non-francophones pour ce type de lexique présent d'une manière significative dans la langue parlée. Certains noms de marques français naturellement compréhensibles pour les locuteurs natifs, peuvent paraître opaques aux locuteurs étrangers. Ceci peut s'expliquer par le contexte culturel qui joue parfois un rôle très important dans la réception du public : alors que certains noms de marques jouissent d'un caractère universel, ou du moins sont communes à plusieurs cultures, d'autres sont profondément inscrits dans un contexte particulier. C'est ce que nous montrerons dans le présent article où nous avons identifié des noms de marques caractéristiques pour la langue-culture slovaque, mais aussi tchèque, par héritage de l'Etat commun tchécoslovaque.

\section{Noms de marques}

\subsection{Aspect marketing}

La problématique des noms de marques, des noms déposés et des noms de produits relève avant tout du domaine du marketing : l'image positive dans le public des noms de marques, favorisant la commercialisation de ses produits est une affaire de marketing. Aussi, dans l'optique du marketing « Une marque constitue un signe ou un ensemble de signes distinctifs (nom, logo, valeurs...) reconnaissable par les consommateurs et créant de la valeur sur un marché pour une entreprise. Elle peut être liée à un producteur (marque propre) ou à un distributeur. Au-delà du produit, une marque est constituée d'un ensemble de valeurs qui créent son identité » (https://www.e-marketing.fr/).

Selon Kapferer (2006), le plus grand spécialiste du domaine des marques, celles-ci sont omniprésentes. Pour le marché, une marque est plus qu'un nom sur un produit : c'est le nom d'un produit qui réussit, dans la mesure où la marque accroît la valeur perçue du produit ou du service. La marque est donc source de rentabilité pour l'entreprise. Une marque ne naît pas, elle devient ce qu'elle est. En marketing, seule la perception compte. Or, il faut du temps pour qu'un signe acquière de la valeur et devienne un signifiant valorisé au point d'influencer les acheteurs, prêts à payer plus cher les produits arborant le signe (https://www.universalis.fr/dictionnaire). Dans cette perspective, Kapferer (in Magne, 2011 : 30) a établi les caractéristiques de l'identité de marque reprises par d'autres auteurs. Celle-ci comprend six facettes: la personnalité, l'univers culturel, le physique (c'est-à-dire un ensemble de caractéristiques objectives), la relation, le reflet, l'image mentale. Magne (2011: 51) soutient l'idée que le langage de la marque constitue un puissant amplificateur de l'image. En effet, la marque se fonde sur un langage visuel et symbolique en mesure de favoriser la perception. Bassani $(2010$ : 9) affirme que, grâce à sa forte valeur symbolique, la marque semble satisfaire à la fois les nouveaux besoins du public et l'exigence des producteurs désirant se différencier et optimiser leur visibilité. C'est donc sur la perception de la marque que se concentre aujourd'hui les entreprises.

\subsection{Aspect linguistique}

$\mathrm{Du}$ point de vue linguistique, Altmanova (2016 : 15) affirme que les noms de marques, comme tout nom propre, sont considérés comme " asémantèmes ", c'est-à dire privés de sens propre, tout en possédant un référent externe. Or, le phénomène de la marque n'est pas récent. Selon Altmanová $(2016: 7,9,11)$, l'histoire de la marque remonte à l'Antiquité. Il est vrai que, de nos jours, son importance ne cesse de grandir. Chacun de nous connaît passivement environ 1500 marques. Les noms de marques entrent dans le lexique général et s'intègrent à la langue de la même manière que tout néologisme formel. Certains noms de marques sont très répandus au niveau mondial. C'est le cas des marques comme Barbie (poupée), Labello (tube de soin pour 
lèvres), Nescafé (café soluble), Pampers (produits pour nourrissons), etc. Galisson $(1998,2000)$ a déjà montré l'intérêt pour les linguistes et didacticiens d'une réflexion sur les marques. Ont suivi des recherches comparatives entre l'approche marketing et l'approche linguistique de la création des noms de marques et de produits (Altmanova 2013, 2016, 2017; Druetta 2008; Fevre-Pernet - Roche, 2005; Laurent, 2010 ; Le Tallec Lloret, 2017 et d'autres).

En français, il existe même des dictionnaires des noms de marques, ce qui n'est pas le cas en slovaque, comme le Dictionnaire des noms de marques courants de R. Galisson et J.-Cl. André (1998: 61) qui fournit un type d'informations qu'on ne trouve ni dans les dictionnaires de langues, monolingues ou plurilingues, ni dans les dictionnaires encyclopédiques, ni dans les banques de données accessibles sur l'internet. L'intention de ce dictionnaire est de montrer (Galisson, $2000: 60$ ) que la culture affirme massivement sa présence dans les types de discours qui n'ont pas pour objet de parler d'elle, c'est-à-dire dans les discours spécialisés ou paraspécialisés. Le locuteur qui parle d'autre chose que de culture parle aussi de culture. Nous partageons l'opinion de Galisson $(2000,59)$ qui affirme que la prolifération des noms de marque est un fait culturel très important, et révélateur de l'omniprésence du fait culturel dans toutes les sphères de la société, notamment dans la vie économique.

Le domaine lexicographique consacré aux noms de marques est l'onomastique commerciale Selon Fèvre-Pernet - Roché (2005:1), ce terme englobe l'étude de l'ensemble des catégories suivantes : les noms de marques, de produits, de gamme, de lignes, de services, mais aussi les noms d'enseignes, et de magasins. Sur le plan juridique, ces noms sont en général des noms déposés. Le nom de marque identifie l'origine, par exemple «Renault», le nom de produit répond à un besoin de nomination, par exemple «Clio» à l'arrière du véhicule identifie le type de voiture/modèle de la marque «Renault». Prenons un autre exemple, celui de «Coca Cola» qui est en même temps un nom de marque et un nom de produit. Pour l'onomastique commerciale, les noms de marques et les noms de produits constituent, linguistiquement, deux classes distinctes. Ces auteurs font la distinction précise entre les noms de marques et les noms de produits en expliquant qu'au début de l'ère industrielle, la marque avait pour fonction première d'identifier l'origine. Aujourd'hui, la marque ne renvoie plus forcément au fabricant, mais, de plus en plus, à une entité abstraite. Cette entité abstraite est cependant chargée de sens ou du moins véhicule un concept, voire un univers que la communication publicitaire se charge de construire et de pérenniser. Druetta (2008 : 174) affirme aussi que les noms de marques sont considérés comme objets linguistiques, culturels et identitaires. Selon Druetta (2008 : 157,160), la création des noms de marques est liée à l'identité nationale et s'appuie sur des mécanismes linguistiques particuliers. La langue est l'une des composantes de base de cette identité, elle est investie d'une fonction symbolique de marqueur d'identité. Petit $(2006: 690,730)$ souligne que la classe des noms de marques déposées est très diversifiée, voire hétérogène. Etant donné le caractère hybride des noms de marques, ils comportent aussi bien des noms propres, des noms communs que des termes techniques, mais sans toutefois posséder pleinement les propriétés du nom propre, du nom commun ou du terme technique. A cette diversité structurelle, correspond une hétérogénéité sémiotique.

Selon Fèvre-Pernet - Roché (2005), deux facteurs principaux concourent à la lexicalisation des noms de marques : la notoriété du produit et la forme que le fabricant a su lui donner (certains se prêtent mieux que d'autres à leur lexicalisation) . Les noms de marques et leurs dérivés contribuent également pour une part importante à la création lexicale. Ainsi, de nombreux néologismes dérivent de noms de marques et s'intègrent à la langue courante mais aussi à la terminologie spécialisée, notamment dans le discours publicitaire (Altmanova, 2016 : 30). Les procédés de la formation des 
noms de marques sont les mêmes que ceux employés pour le lexique général, surtout dans le cas des noms de produits. La plateforme de veille néologique Neoveille permet de rendre compte de ces processus. Comme nous l'explique Cartier (2016), la plateforme de veille néologique s'appuie encore aujourd'hui sur la méthode traditionnelle de l'expertise et de l'intuition linguistique qui reste la méthode préférentielle de tout travail sur la néologie, au moins du point de vue de l'analyse des nouvelles formes et des nouveaux sens.

Pour la recherche et le suivi des néologismes, parmi les systèmes génériques, il faut citer deux outils régulièrement utilisés par les linguistes pour effectuer une veille néologique : les moteurs de recherche généraux ou spécialisés, et l'application Google Trends. Il est nécessaire d'effectuer des recherches d'attestations néologiques, dans notre cas des marques connues, des nouvelles marques et de leurs dérivés.

L'analyse du corpus que nous avons constitué à partir de noms de marques français et slovaques met en évidence des différences d'ordre culturel entre les deux languescultures modélisées par Schmitt (2015: 113-114). Ces différences culturelles surgissent avant tout au cours du processus de traduction, comme nous avons pu le remarquer en cherchant les équivalents slovaques de noms de marques français. Koláŕiková - Mudrochová (2019) ont fait la même observation à propos des noms déposés qui représentent un phénomène linguistique très intéressant, surtout dans le contexte de la traduction.

\section{Le corpus de noms de marques et son analyse}

Le corpus présenté ici, servant à répertorier des noms de marques, a été constitué pour l'essentiel à partir de forums de spécialistes et de blogs. Nous nous intéressons avant tout à la langue parlée. Parmi les noms de marques répertoriés, on rencontre souvent la forme tronquée $($ MacDonald $=$ Macdo $)$. C'est un phénomène linguistique qu'on ne peut ignorer. On peut l'observer aussi en slovaque (Nescafé = neska). Selon Le Tallec Lloret (2017), «la troncation est un indice fiable sur le degré de pénétrabilité d'un nom de marque dans le langage courant, et, de fait, de son succès - son nom étant suffisamment connu et reconnu pour pouvoir subir dans l'échange langagier une coupe ne faisant pas obstacle à la bonne compréhension entre le locuteur et son interlocuteur»!.

Nous avons réparti les noms de marques de notre échantillon en quatre groupes. Le premier contient des noms de marques françaises qui n'ont aucun équivalent en slovaque. Le deuxième groupe contient des noms de marques françaises possédant des équivalents slovaques facilement identifiables. Le troisième groupe contient des noms de marques slovaques sans équivalents français. Le dernier groupe contient des noms de marques slovaques et tchèques hérités de la Tchécoslovaquie et partagés aujourd'hui encore par la Slovaquie et la République tchèques.

Pour présenter les résultats de notre analyse, nous précéderons de la manière suivante. Nous donnerons d'abord l'exemple français, avec éventuellement sa variante tronquée, en présentant, le cas échéant, son équivalent slovaque. Nous fournirons, ensuite, entre parenthèses, quelques informations utiles sur la marque et son origine. Les noms des marques seront à chaque fois suivis des énoncés authentiques d'où nous les avons tirés et seront accomagnés des sources. Enfin, entre crochets, nous donnerons la traduction en français de l'exemple slovaque pour mieux faire apparaitre les différences.

\subsection{Le premier groupe : des noms de marques français sans équivalent en slovaque}

XLinguae, Volume 12 Issue 1XL, January 2019, ISSN 1337-8384, eISSN 2453-711X 
Il s'agit de noms de marques français qui n'existent pas en slovaque.

(1) Kleenex $=$ sans équivalent en slovaque

(Les mouchoirs en papier furent baptisés du nom de marque Kleenex ${ }^{\circledR}$ et commercialisés pour la première fois en 1924. De nos jours, ce nom de marque désigne un mouchoir en papier jetable, quelle que soit la marque).

Tiens, passe-moi un kleenex. Elle se moucha.

https://books.google.sk/books?

Se moucher dans un kleenex.

https://www.notrefamille.com/dictionnaire/definition/kleenex/

(2) Bic (stylo à bille en matière plastique, d'aspect rudimentaire, bon marché, jetable)

(Le premier « vrai » stylo à bille date des années $30 \mathrm{du} 20^{\mathrm{e}}$ siècle. Il a vu le jour en Argentine grâce à un Hongrois, Laszlo Biro, qui le commercialisa sous le nom de Birome. Mais, c'est en 1952 que l'utilisation du stylo à bille fut démocratisée grâce au baron Marcel Bich, qui, après avoir racheté la licence Biro, lança le stylo à bille Cristal sous sa marque, raccourcie de son propre nom et devenue universelle : Bic.

Combien de pages peut-on écrire avec un stylo Bic?

http://www.bu2z.com/question-con-combien-pages-ecrire-stylo-bic

\subsection{Le deuxième groupe : des noms de marques français avec leurs équivalents en slovaques}

Les noms de marques communs aux deux langues différent par leurs formes adoptées dans l'usage. En français, la troncation est très fréquente (Le Tallec Lloret : 2017). On retrouve le même procédé en slovaque auquel il faut ajouter la forme argotique.

\section{(3) McDonald $=$ Macdo/Mcdo $=$ Mekáč}

(McDonald's : chaîne de restauration rapide américaine)

J'ai mangé McDonald pendant 2 ans et cet aliment a tué mes abdos.

http://laroutedelaforme.fr/manger-mcdo-pendant-2-ans/

Comme bon nombre de jeunes, j'ai atterri au McDo à cause d'un .... ils étaient plutôt détendus du gland puisque j'ai mangé macdo non stop (...).

http://www.madmoizelle.com/travailler-mc-donalds-61777

Pourquoi on continue malgré tout d'aller au McDo.

http://www.elle.fr/Elle-a-Table/Les-dossiers-de-la-redaction/News-de-la-

redaction/Pourquoi-on-continue-malgre-tout-d-aller-au-McDo-3659696

Manger sain fast food (...) Et en effet, en cherchant bien, on finit par dénicher chez McDo un petit onglet (...) Je mange quoi au restaurant en période de régime?

https://www.marieclaire.fr > ... > Nutrition $>$ Conseils nutrition

En slovaque :

Mekáč sa trápi ako už dávno nie. Pomôct' má nový šéf.

14.02.2015 www.trend.sk

[Mekáč est en difficultés comme jamais. Il peut s'en sortir avec un nouveau chef.] 
(4) Nescafé $($ café soluble $)=$ nes $=$ nescafé $/$ neska

(Café en poudre soluble; par métonymie, boisson préparée avec cette poudre, nom de marque déposée par la Société Nestlé au greffe du tribunal de commerce de la Seine en 1939).

La machine Barista promet de faire mousser le Nescafé (...).

https://www.24heures.ch/vivre/machine...nescafe/.../14896101

En français, on utilise la forme tronquée nes.

Adepte du café en capsules, j'ai eu l'occasion d'acheter le café NES afin de l'emporter en vacances très pratique à utiliser, préparation rapide pour un résultat parfait, le café est de qualité sans amertume. Très bon rapport qualité prix.

https://www.croquonslavie.fr/marques/produits-nestle/nescafe/nescafe-nes-cafesoluble-boite-100g

En slovaque, il existe une forme tronquée neska qui est très répandue et plus utilisée dans la langue parlée et la communication courante que la forme de base nescafé.

Ahoj, aj ja pijem nesku ráno a sem tam si dám aj poobede. https://mamaaja.sk/diskusie/babatka/dojcenie/diskusia-1092

[Salut, moi aussi, je bois du «neska » le matin et parfois, j'en prends aussi l'aprèsmidi.]

(5) Pampers $($ produits pour nourrissons $)=$ Pampes $/$ pampersky

(La marque Pampers est une marque américaine de produits pour nourrissons commercialisés par Procter \& Gamble depuis 1971)

Ce qui a fait le succès de Pampers est justement de toucher directement les mères (...).

https://www.etudier.com/dissertations/Strat\%C3\%A9gie-Qualit\%C3\%A9-Pampersp-g/357563.html

Dôrazne žiadame nedávat’ pampersky a plienky!

https://www.hronskekosihy.sk/ako-separovat.html

[Nous vous demandons impératiment ne pas y mettre de pampers et de couches!]

(6) Coca $($ Coca Cola $)=$ kola

(Le Coca Cola est une marque déposée de The Coca-Cola Company, créé aux ÉtatsUnis en 1887, enregistré en Slovaquie en 1992).

Je suis un ancien gros buveur de coca.

forum.doctissimo.fr/nutrition/alimentation-sante/chaque-dangereux-docteursujet_157156_1.htm

Konzumácia koly oslabuje ženám kosti... v bedrách ako ženy, ktoré mesačne vypili menej ako jednu kolu. https://www.24hod.sk/konzumacia-koly-oslabuje-zenam-kosticl20504.ht

[La consommation de coca affaiblit les os des femmes (...) dans les reins des femmes qui ont bu moins d'un coca par mois.]

\subsection{Le troisième groupe : les noms de marques slovaques}

XLinguae, Volume 12 Issue 1XL, January 2019, ISSN 1337-8384, eISSN 2453-711X 
Il s'agit d'exemples de la langue et de la culture slovaques sans équivalent en français.

(7) Demänovka

(Demänovka, liqueur slovaque à base de plantes, fabriquée depuis 1954)

Matka ma hladkala po ruke a z vd'ačnosti mi naliala ešte jednu demänovku. Priznám sa, bol som dost' pomýlený.

https://www.google.com/search?client=firefox-

[Maman m'a caressé la main et, pour me remercier, m'a versé encore une demänovka. J'avoue avoir été assez confus.]

(8) Rumba = barre de chocolat

(Rumba, barre de chocolat, produit par une chocolaterie créée à Bratislava en 1896 par les frères Stolwerk, aujourd'hui, l'entreprise Figaro)

Čokoláda je vynikajúca, ale opatrne ako so všetkými dobrými vecami (...) ale čokoládu si občas dám (...) taká malá rumba bodne.

30.9.2018 https://ekonomika.sme.sk/.../Cokoladu-jedia-Slovaci-kazdy-den-castejsienez-Cesi.html

[Le chocolat est délicieux, mais faites attention comme avec toutes bonnes choses (...) parfois, je mange du chocolat (...) un petit rumba, ça tombe bien.]

\section{(9) Indulona}

(Indulona, crème mains fabriquée par l'entreprise Slovakofarma à Hlohovec en Slovaquie depuis 1948)

Indulona je super, hlavne modrá, a to niektorí mudrci chceli po 89 roku zrušit' jej výrobu.

25. 2. 2010 - https://blog.sme.sk/diskusie/1644194/1/Nobelovka-pre-Indulonu.html

[Indulona est super, surtout la bleue, et dire que certains voulaient supprimer sa production après 89.]

(10) Marína

(Marína - biscuits fabriqués par l'entreprise Ružový mlyn à Piešt’any en Slovaquie depuis 1948)

Cez den̆ pomedzi jedlá pije MM na požiadanie, ale dost' málo,neviem odhadnut' kol'ko. Viac MM pije v noci (...) Diet'a v tomto veku nemá čo dostávat' marína keks! https://mamaaja.sk/poradne/poradna-dojcenia-a-vyzivy/strana-29 [A cet âge, il n'y a aucune raison de donner à un enfant des biscuits marína !]

\subsection{Le quatrième groupe : des noms de marques slovaques et tchèques}

Il s'agit d'exemples de marques slovaques et tchèques de l'époque tchécoslovaque (quand les Slovaques et Tchèques partageaient un Etat commun : la Tchécoslovaquie) sans équivalent en français. Ce groupe contient le plus de noms de marques.

(11) Becherovka

(Becherovka, liqueur tchèque à base de plantes, fabriquée depuis 1807)

(...) dlhšie sa zamyslel, nalial si ešte jednu lepkavú becherovku(...). “

http://debata.pravda.sk/debata/blog-anaj-318-2011-12-06-co-sa-judas-nachodi-kymvyjde-na-psi-tridsiatok/?view_mode=vlakna\&ordering=od_najnovsieho\&strana=7 
$[(\ldots)$ il a réfléchi un moment, il s'est versé encore une becherovka un peu trop sucrée (...).]

Začal som robit’ drepy a cvičit', dal som si čaj a becherovku.

https://lav.sk/ovladnite-svoj-imunitny-system-metoda-wima-hofa-na-vlastnej-kozi/ [J'ai commencé à faire des pompes, de la gymnastique, j'ai pris un thé et une becherovka.]

(12) Kofila

(chocolat Kofila fabriqué depuis 1932 par Orion)

„(...) že ja som sa tak delila aj o kofilu (...)“

03.06.2012 wwb.dogforum.sk/viewtopic.php $? f=80 \& t=206 \&$ start $=15930$

[(...) j'ai tout partagé, même un kofila (...)]

(13) Botasky

(marque de chaussures de sport Botas fabriquées en République tchèque depuis1949)

$V$ e-shope ROBEL.sk máme značkové botasky, svetových výrobcov. Zvučné značky našich botasiek zaručujú pohodlnost' a kvalitu.

https://www.robel.sk/znackove-botasky

[Dans l'e shop Robel.sk, nous vendons des botasky de marque de producteurs mondiaux (...).]

(14) Bat'ovky

(chassures en toile de la marque Bata, société A. \& T. Bat'a fondée en 1894 à Zlín en Tchécoslovaquie, chaussures fabriquées aussi à Partizanske en Slovaquie)

Bat’a by zaplakal: V Partizánskom si bat’ovky nekúpite, úder prišiel z Číny. https://www.pluska.sk/plus-7-dni/domov/bata-zaplakal-partizanskom-si-batovkynekupite-uder-prisiel-z-ciny.html (22.3. 2016)

[Bata en pleurerait : vous n'achetez plus batovky à Partizanske, le coup est venu de Chine.]

\section{(15) Jar}

(liquide vaisselle, détergent, fabriqué depuis 1955 par l'entreprise Rakona à Rakovník en République tchèque)

Dôkladne umyjeme pomaranče (ja umývam jarou $v$ teplej vode a dobre opláchnem.) https://www.zenyvmeste.sk/recept--pomarancova-polievka [(...) laver bien les oranges, je les lave avec jar à l'eau chaude.]

Le nom de marque Jar est devenu un nom commun, utilisé aussi pour une autre marque slovaque PUR ou d'autres marques de liquide vaisselle.

(16) Škoda Auto = la Skoda

(Les origines de Škoda remontent à 1862 quand le Tchèque Emil Škoda rachète l'usine métallurgique où il travaillait depuis dix ans et en fait l'une des plus grandes de l'époque. A la fin de la Première Guerre mondiale, Škoda se lance dans la production automobile. Depuis 1991, l'entreprise appartient au constructeur automobile Volkswagen Group).

XLinguae, Volume 12 Issue 1XL, January 2019, ISSN 1337-8384, eISSN 2453-711X 
Milovníci starých škodoviek si prídu na svoje. Polícia zverejnila fotografie, na ktorých je zachytená poctivá práca šikovného Jakuba.

https://www.topky.sk/cl/13/1726891/FOTO-Jakub-prerobil-staru-skodovku-na-

paradnu-masinu--Neuverite--co-sa-s-nou-da-robit

[Les amateurs de vieilles skodovka seront contents. La police a publié des photos où on peut voir le travail minitieux de Jakub qui est très doué.]

Môj otec dával na zimu škodovku na tehly.

http://m.motoride.sk/?P=phorum\&tema=poradna\&tid=74477

[En hiver, mon père mettait la skodovka sur des briques]

Les exemples slovaques (et tchèques) des noms de marques sont la preuve que les bonnes marques reflétent la bonne qualité de leurs produits et c'est pourquoi elles se sont lexicalisées assez rapidement (Marina, Rumba, Indulona, Bat'ovky, Botasky...), et ceci déjà sous le régime communiste. Avec l'économie de marché en 1989 et la mondialisation, de nouvelles marques ne cessent d'apparaître, à titre d'exemple Mekáč, Pampers, Labello, etc. Ces derniers exemples sont intéressants pour la recherche contrastive. Dans certains cas, le nom de la marque étrangère est adopté tel quel, sans modification (Labello), d'autres, auxquels on a simplement ajouté un suffixe, comme - ky (Pampersky), restent facilement identifiables. Par contre, l'exemple de Mekáč n'est pas reconnaissable pour un étranger. Il s'agit d'une forme argotique tronquée.

Cette analyse nous montre que langue et culture sont étroitement liées dans le processus de naturalisation des noms de marques dans les situations de communication courante, non seulement en ce qui concerne les noms de marques communes en français et en slovaque, mais encore davantage dans le cas des noms de marques slovaques et tchèques, profondément ancrés dans le patrimoine culturel (tchèco)slovaque.

\section{Conclusion et perspectives}

Bien connue du marketing, l'étude des noms de marques l'est beaucoup moins du point de vue linguistique, sémantique et culturel. Les premiers résultats de notre recherche contrastive concernant le recensement et l'étude des noms de marques français et slovaques nous montrent donc tout l'intérêt scientifique que représente ce domaine socioculturel pour la langue et la culture, en particulier pour la langueculture slovaque. Parmi les nombreuses perspectives offertes par ce domaine de recherche encore peu exploité figure, par exemple, la question de la création lexicale dont les applications dans l'enseignement des langues, notamment l'enseignement des langues sur objectifs spécifiques, et en traductologie sont évidentes, particulièrement en ce qui concerne les compétences interculturelle et lexicale par la familiarisation aux néologismes. Ce domaine mérite donc d'être élargi à d'autres langues. Est également envisageable un élargissement de l'étude comparée des noms de marques au profit de l'intercompréhension interlinguistique, en premier lieu en intercompréhension des langues romanes, mais aussi pour d'autres groupes de langues, germaniques ou slaves.

\section{Bibliographic references}

ALTMANOVA, J. 2016. Du nom depose au nom commun. Neologie et lexicologie en discours. Milano :EDUCatt. ISBN 978-88-6780-037-7.

ALTMANOVA, J. 2017. Les derives des noms de marques et de produits, In : Christophe Gerard (ed.), La creation en situation. Textes, Genres, Cultures (sous presse). 
BASSANI, M. - BEN YOUSSEF, K. - MAGNE, S. - SBALCHIERO, S. 2010. Brand Design - construire la personnalité d'une marque gagnante, $2^{\text {ème }}$ édition, Paris : Éditions De Boeck. ISBN 978-2804162474.

CARTIER, E. 2016. Neoveille, système de repérage et de suivi des néologismes en sept langues. In Neologica, 10, Revue internationale de néologie, pp.101-131. langues Available online

https://www.researchgate.net/publication/311585679_Neoveille_systeme_de_reperag e_et_de_suivi_des_neologismes_en_sept_langues

DRUETTA, R. 2008. Les noms de marque et de produit comme marqueurs identitaires. In: Ela. Etudes de linguistique appliquée. Paris : Klincksieck. 2008/2 n ${ }^{\circ}$ 150 , pp. 157-175. ISSN 0071-190X, ISBN 9782252036501. https://www.cairn.info/revue-ela-2008-2-page-157.htm.

FEVRE-PERNET, Ch. - ROCHE, M. 2005. Quel traitement lexicographique de l'onomastique commerciale ? Pour une distinction Nom de marque/Nom de produit. Corela [En ligne], HS-2 2005, mis en ligne le 02 décembre 2005, consulté le 30 juillet 2018. Available online : http://journals.openedition.org/corela/1198; DOI : 10.4000/corela.1198.

GALISSON, R. - ANDRÉ, J.-C. 1998. Dictionnaire des noms de marques courants : essai de lexiculture ordinaire. Paris: Didier Érudition. ISBN 978-2864603405.

GALISSON, R. 2000. La pragmatique lexiculturelle pour acceder autrement, à une autre culture, par un autre lexique. In: Mélanges, 2000, n. 25, pp. 47-73. ISSN papier :

0077-2712.

ISSN électronique : 1952-4250.

KAPFERER, J. -N. 2006. FAQ La Marque - La marque en questions : reponses d'un specialiste. Paris: Dunod. ISBN 2-10-049127-X.

KAPFERER, J. -N. Marque, marketing. Encyclopædia Universalis. Available online : http://www.universalis.fr/encyclopedie/marque-marketing/.

KOLARIKOVA, D. - MUDROCHOVA, R. Les noms deposes issus du Petit Robert et leurs equivalents en tcheque. In: Jana Altmanova, Gabrielle Le Tallec (éds). Lexicalisation de l'onomastique commerciale. Créer, diffuser, intégrer. Naples (à paraître).

LARRIEU, J. 2011. Les métamorphoses de la marque. Toulouse: Presses de l'Universite Toulouse 1 Capitole. LGDJ. ISBN 978-2361700317.

LAURENT, B. 2010. Nom de marque, nom de produit : semantique du nom depose (Sémantiques). Paris: L'Harmattan. ISBN 9782296123885.

LE TALLEC LLORET, G. 2017. Lexicalisation et banalisation des noms de marque : le cas juridique de Placoplatre ${ }^{\circledR}$ et Placo ${ }^{\circledR}$. In: Colloque international LEXIMARQ Lexicalisation de l'onomastique commerciale - créer, diffuser. <hal-01875599>. https://hal.archives-ouvertes.fr/hal-01875599.

MAGNE, S. 2011. Marque et marketing : construire la personnalite d'une marque In: Larrieu Jacques (ed.). Les métamorphoses de la marque. 2011. Toulouse: Presses de l'Universite Toulouse 1 Capitole. LGDJ - Lextenso Editions. pp. 37-53. ISBN 9782361700317. Available online : https://books.openedition.org/putc/2601.

PETIT, G. 2006. Le nom de marque déposée : nom propre, nom commun et terme. In

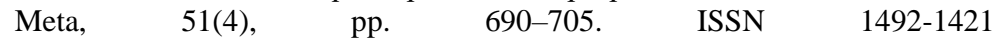
(numérique).https://doi.org/10.7202/014335ar.

SCHMITT, F. Les cultures francaise et slovaque. Analyses comparatives de representations sociales. Louvain-la-Neuve : Editions EME. ISBN 978-2-8066-32517.

Words: 4356

Caracters: 25709 (14,28 standard pages)

XLinguae, Volume 12 Issue 1XL, January 2019, ISSN 1337-8384, eISSN 2453-711X 227 
Assoc. prof. Ludmila Meskova, PhD.

Department of European cultural studies

Faculty of Arts, University Matej Bel, Banska Bystrica

Tajovskeho 40, 97401 Banska Bystrica

Slovakia

ludmila.meskova@umb.sk

Assist. prof. Jana Pecnikova, PhD.

Department of European cultural studies

Faculty of Arts, University Matej Bel, Banska Bystrica

Tajovskeho 40, 97401 Banska Bystrica

Slovakia

jana.pecnikova@umb.sk 